\title{
Incidence of gastric cancer among patients with gastric precancerous lesions: observational cohort study in a low risk Western population
}

\author{
Huan Song, ${ }^{1}$ Isabella Guncha Ekheden, ${ }^{1}$ Zongli Zheng,, Jan Ericsson,, ${ }^{2}$ Olof Nyrén,, Weimin Ye ${ }^{1}$
}

${ }^{1}$ Department of Medical

Epidemiology and Biostatistics,

Karolinska Institutet, 17177

Stockholm, Sweden

2Department of Pathology, Karolinska Hospital, Sweden

Correspondence to:

WYeweimin.ye@ki.se

Additional material is published online only. To view please visit the journal online (http://dx.doi. org/10.1136/bmj.h3867)

Cite this as: BMJ 2015;351:h3867 doi: 10.1136/bmj.h3867

Accepted: 3 July 2015

\section{ABSTRACT}

\section{OBJECTIVE}

To accurately measure the incidence of gastric cancer among patients with gastric precancerous lesions, and to quantify the excess incidence in comparison with people with normal mucosa on endoscopy and a general population.

\section{DESIGN}

Population based cohort study.

SETTING

Population of Sweden using data from its national disease registers.

\section{PARTICIPANTS}

405172 patients who had gastric biopsy samples taken for non-malignant indications between 1979 and 2011.

\section{MAIN OUTCOME MEASURES}

Incidence of gastric cancer, reported separately for patients with different mucosal changes in biopsy samples. Standardised incidence ratios provided estimation of the relative risk, using the general Swedish population as reference; and hazard ratios were derived from Cox regression modelling for internal comparisons with patients with normal gastric mucosa.

RESULTS

After excluding the first two years of follow-up, 1599 cases of gastric cancer were identified. The annual crude incidence of gastric cancer was $20 \times 10^{-5}$ for those in the normal mucosa group (standardised incidence ratio 1.0$), 42 \times 10^{-5}$ for those with minor changes (1.5), $59 \times 10^{-5}$ for the gastritis group (1.8), $100 \times 10^{-5}$ for the

\section{WHAT IS ALREADY KNOWN ON THIS TOPIC}

Gastric cancer is preceded by a series of precancerous lesions, but the risk of gastric cancer in patients with such lesions is not well quantified

Only one study provided follow-up data from a nationwide biopsy cohort, albeit with imputed censoring information

In that study the excess incidence of gastric cancer among all patients with premalignant gastric lesions, especially in the first few years of follow-up, was considerable

\section{WHAT THIS STUDY ADDS}

Our data predict that about 1 in 256 people with normal mucosa, 1 in 85 with gastritis, 1 in 50 with atrophic gastritis, 1 in 39 with intestinal metaplasia, and 1 in 19 with dysplasia will develop gastric cancer within 20 years after gastroscopy If repeated biopsy showed a change-upward or (more noteworthy) downward in the Correa's cascade-compared with the initial grouping, this seemed to have prognostic significance

Further cost-benefit deliberations are needed to guide policy making on long term endoscopic surveillance of premalignant gastric lesions in low risk Western populations atrophic gastritis group (2.8), $129 \times 10^{-5}$ for the intestinal metaplasia group (3.4), and $263 \times 10^{-5}$ for the dysplasia group (6.5). Cox regression modelling confirmed that excess risks increased monotonically with progressive severity of gastric lesions, with the highest hazard ratio of 10.9 (dysplasia versus normal mucosa, $95 \%$ confidence interval 7.7 to 15.4 ). The increased incidence was stable throughout the follow-up period, and the gaps between cumulative incidence curves grew continuously.

\section{CONCLUSIONS}

Among patients who undergo gastroscopy with biopsy for clinical indications, approximately 1 in 256 with normal mucosa, 1 in 85 with gastritis, 1 in 50 with atrophic gastritis, 1 in 39 with intestinal metaplasia, and 1 in 19 with dysplasia will develop gastric cancer within 20 years. These numbers, along with costbenefit analyses, should guide future surveillance policies for these particular patient groups.

\section{Introduction}

The successive progression from chronic non-atrophic gastritis, by way of atrophic gastritis and intestinal metaplasia, to dysplasia, known as Correa's cascade, ${ }^{12}$ is widely recognised as a common route to the intestinal type of non-cardia gastric cancer. ${ }^{3}$ Helicobacter pylori infection has been generally accepted as the initiator of this cascade, ${ }^{45}$ and epidemiological data support its role as the most important risk factor for gastric cancer. ${ }^{67}$ However, follow-up data in humans on absolute risks associated with having these precancerous lesions are inconsistent. ${ }^{8-10}$ For instance, reported average risks of gastric cancer among patients with dysplasia vary from $0 \%$ to $73 \% .^{11}$ This has led to uncertainties about the clinical management. ${ }^{12}$

As in other Western countries, the incidence of gastric cancer in Sweden has declined noticeably. The downward trend can date back to 1940, although it has slowed down in recent decades. ${ }^{13}$ However, a surprising increase among young US white people aged 25-39 years has been reported, ${ }^{14}$ and we have also noted an increasing trend of atrophic gastritis among adults aged 35-44 years in Sweden, ${ }^{15}$ which implies that the falling trend of gastric cancer might be reversed in the near future. Taking advantage of the complete computerised registration of biopsy investigations and specimens examined at all Swedish pathology departments, we conducted a nationwide cohort study of all registered Swedish patients with stomach biopsy samples taken at endoscopy units. We aimed to accurately measure the incidence of gastric cancer among patients with minor changes, chronic 
non-atrophic gastritis, atrophic gastritis, intestinal metaplasia, and dysplasia at baseline, and to quantify the excess incidence in comparison with patients with normal mucosa on endoscopy and the matched Swedish general population.

\section{Methods}

Databases

All pathology departments in Sweden hold computerised disease registers. The first one was established in 1979 and the last in 1998. From these registers we compiled a cohort consisting of all patients registered with a gastroscopic stomach biopsy for non-malignant indications up to 31 December 2011. The 24 pathology departments provided the patients' national registration numbers (unique identifiers for all Swedish residents) along with data on date, age, sex, and pathological-anatomical diagnosis using the systematised nomenclature of medicine morphology (SNOMED M) codes. ${ }^{16}$ Each record contained information about one examination. Therefore patients could have several records in the compiled database.

In the research database we compiled 613637 records relating to 437473 people who had undergone at least one endoscopic examination with stomach biopsy. We linked the database to the nationwide and 98\% complete $^{17}$ cancer register using the national registration numbers as identifiers, where all incident cases of gastric cancer were identified. Further cross linkages with the death register, patient register, and emigration register provided the necessary information for eligibility assessment and censoring.

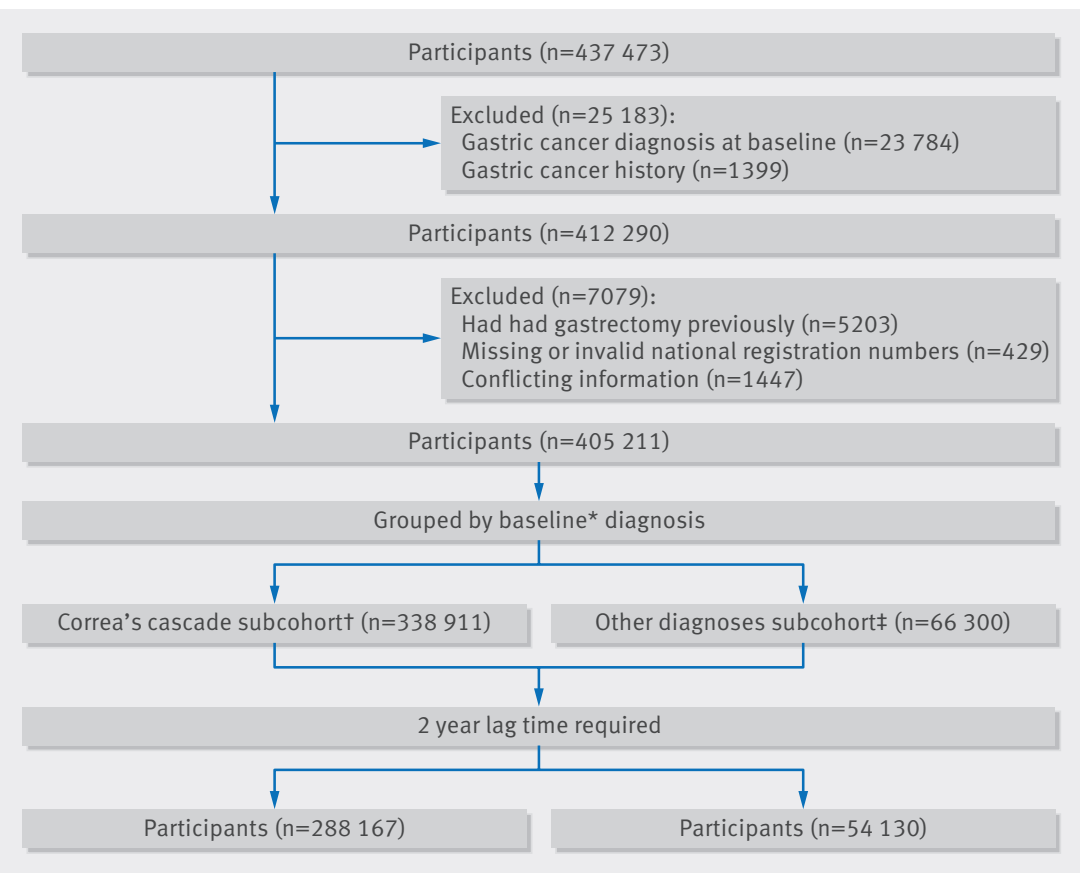

Fig 1 | Study design for stomach biopsy cohort in Sweden (1979-2011). *Baseline defined as first biopsy identified in database. When multiple diagnoses were present, the most severe one was selected. tCodes for normal, minor mucosal change, gastritis, atrophic gastritis, intestinal metaplasia, and dysplasia. ¥Codes for "other diagnoses" (see supplementary table for specific codes)

\section{Study design}

For each participant we defined the date of cohort entry (baseline) as the date of first biopsy identified in the database. After exclusion of patients with a new ( $n=23784)$ or previous ( $\mathrm{n}=1399)$ diagnosis of gastric cancer at baseline, we kept in our database 412290 patients who underwent biopsy for non-malignant indications. We further excluded those who had previously undergone gastric resection or gastrectomy $(n=5203)$, had missing or invalid national registration numbers $(n=429)$, or had conflicting information (died or emigrated before baseline biopsy, $\mathrm{n}=1447$ ). Finally, 405211 eligible patients remained in the cohort, of whom $21.1 \%$ had at least one repeat endoscopy with biopsy result documented. We continued follow-up until the occurrence of gastric cancer, date of gastric resection, migration out of Sweden, death, or the end of follow-up (31 December 2011), whichever occurred first.

Gastric mucosal status at baseline-We grouped the cohort members by their SNOMED M diagnosis at baseline (see supplementary table). Our main focus was on Correa's cascade, with the groups (in order of progression): normal, minor mucosal changes, non-atrophic chronic gastritis, atrophic gastritis, intestinal metaplasia, and dysplasia. However, to prevent the possible effects of other clinical diagnoses on measurements for gastric cancer risk, we first identified 66300 patients whose main findings were of uncertain relevance to Correa's cascade lesions and to development of gastric cancer (in order of severity: cellular degeneration/infiltration or abnormal karyotype, reactive gastropathy, atypia, hyperplasia or hypertrophy, metaplasia, polyps, and benign tumour). We analysed these groups separately under the subheading "other diagnoses" (fig 1). If more than one of the above findings was recorded, we grouped the patient as having the most severe one. For the remaining 338911 patients (the Correa's cascade subcohort) with normal gastric mucosa, minor changes, or lesions in Correa's cascade, we determined the baseline group by the diagnostic code that indicated the most advanced lesion.

Outcome ascertainment-We identified cases of gastric cancer through linkage with the Swedish cancer register, using the seventh version of the international classification of diseases (ICD-7) code 151. In subanalyses, we divided gastric cancer into cancer of the gastric cardia (code 151.1) and non-cardia gastric cancer (except code 151.1). Patients who underwent gastric resection for non-malignant indications during the follow-up were censored on the date of the operation. Gastric resections were ascertained in the patient register. For major upper gastrointestinal surgery, the completeness of the Swedish patient register is deemed to be well over $90 \%{ }^{18}$

\section{Statistical analysis}

We calculated the standardised incidence ratio (the ratio of the observed to the expected number of cases with newly diagnosed gastric cancer) with its 95\% confidence intervals to estimate the relative risk for each exposure group, using the general Swedish population as reference. The expected number of cases was calculated by multiplying the observed number of person years by age (five year strata), sex, and calendar 
year specific incidence rates derived from the entire Swedish population. Further analyses stratified by sex and follow-up duration were also conducted. We used the Nelson-Aalen method to estimate the cumulative incidence of total gastric cancer among those with different baseline diagnoses.

A peak of gastric cancer incidence invariably observed shortly after a first gastric biopsy is principally made up of symptomatic cancers that were overlooked at initial endoscopy, reflecting possibly country specific effectiveness of gastroscopic services. Therefore we chose to start follow-up when this peak had passed. The peak can be visualised by the "interval based method"19 presented in supplementary figure 1, which illustrates the calculated standardised incidence ratios for several disjoint time intervals close to entry into the cohort. Extremely high standardised incidence ratios were observed in the first 24 months of follow-up. Thus in our main analyses we discarded the first two years of observation and outcomes detected during this period.

For internal comparisons within the Correa's cascade subcohort, we evaluated the association between gastric mucosal status and risk of gastric cancer using hazard ratios with 95\% confidence intervals, derived from the Cox proportional hazards regression model. We used attained age as underlying time scale, and adjusted for sex, and stratified by pathology department. The proportional hazards assumption was checked graphically and by Schoenfeld's partial residuals; neither of them revealed any indication of violation of this assumption.

Lastly, among 60727 patients with more than one eligible biopsy record (biopsy sample taken within two years of a gastric cancer diagnosis were disregarded) in Correa's cascade subcohort, we performed supplementary estimations of standardised incidence ratios for each baseline histology group after stratification according to the direction of any change in classification prompted by the histological re-evaluations. Thus, within each baseline histology group, those with more advanced lesions in re-endoscopies were grouped into the "progression" stratum; correspondingly, those with less advanced lesions were grouped into the "regression" stratum, and all others into the "no change" stratum.

We considered a P value of less than 0.05 to be statistically significant. All analyses were conducted in SAS statistical software, version 9.3 (Cary, NC).

\section{Patient involvement}

No patients were involved in setting the research question or the outcome measures, nor were they involved in the design and implementation of the study. There are no plans to involve patients in dissemination.

\section{Results}

After excluding the first two years of follow-up, 288167 patients remained in the Correa's cascade subcohort. They accrued 2381032 person years at risk (table 1). Mean age at entry was 56 years. Those with more advanced lesions tended to be older than those with less advanced ones. While there was a predominance of women overall (male: female 1:1.24), men were over-represented in the dysplasia group (male: female 1:0.95). The average duration of follow-up was around 10 years, similar for most mucosal histology groups, except for the intestinal metaplasia group (7.9 years). In the "other diagnoses" subcohort, 54130 patients remained after the two year lag. They accumulated 381603 person years at risk (table 1). The mean age at entry varied between 53.2 years (reactive gastropathy) and 65.2 years (non-intestinal metaplasia). Male predominance was seen only in the atypia group.

During follow-up, 1599 participants had a diagnosis of gastric cancer-1388 with non-cardia gastric cancer and 211 cardia gastric cancer. Table 2 shows the distribution of gastric cancer cases across mucosal histology groups,

\begin{tabular}{|c|c|c|c|c|c|}
\hline Mucosal status at baseline* & No of participants & $\begin{array}{l}\text { Mean (SD) age at } \\
\text { entry (years)t }\end{array}$ & $\%$ male & $\begin{array}{l}\text { Mean (SD) } \\
\text { follow-up (years) }\end{array}$ & $\begin{array}{l}\text { Accumulated } \\
\text { person yearst }\end{array}$ \\
\hline \multicolumn{6}{|l|}{ Correa's cascade: } \\
\hline Normal & 81174 & $47.7(19.9)$ & 40.5 & $9.7(5.7)$ & 621359 \\
\hline Minor mucosal change & 11571 & $57.5(18.5)$ & 43.8 & $10.2(6.6)$ & 95437 \\
\hline Gastritis & 167521 & $58.3(18.2)$ & 46.5 & $10.7(6.2)$ & 1463788 \\
\hline Atrophic gastritis & 14285 & $60.3(18.5)$ & 41.3 & $10.1(6.3)$ & 115583 \\
\hline Intestinal metaplasia & 11530 & $66.1(14.7)$ & 47.8 & $7.9(4.6)$ & 68122 \\
\hline Dysplasia & 2086 & $65.5(14.9)$ & 51.3 & $10.0(6.0)$ & 16743 \\
\hline Overall & 288167 & $55.7(19.3)$ & 44.5 & $10.3(6.1)$ & 2381032 \\
\hline \multicolumn{6}{|l|}{ Other diagnoses: } \\
\hline $\begin{array}{l}\text { Cellular degeneration/infiltration or } \\
\text { abnormal karyotype }\end{array}$ & 826 & $60.1(18.8)$ & 50.9 & $9.4(5.7)$ & 6120 \\
\hline Reactive gastropathy & 9130 & $53.2(17.5)$ & 37.3 & $5.9(2.9)$ & 35699 \\
\hline Atypia & 5124 & $63.9(14.5)$ & 54.3 & $11.4(6.8)$ & 48254 \\
\hline Hyperplasia or hypertrophy & 12392 & $60.9(16.5)$ & 40.8 & $9.1(5.3)$ & 87853 \\
\hline Metaplasia (not intestinal metaplasia) & 9883 & $65.2(14.7)$ & 49.4 & $10.3(5.7)$ & 82399 \\
\hline Polyps & 13935 & $61.7(14.3)$ & 36.3 & $9.3(5.5)$ & 101289 \\
\hline Benign tumour & 2840 & $62.3(16.1)$ & 35.2 & $9.0(5.6)$ & 19989 \\
\hline Overall & 54130 & $60.9(16.1)$ & 41.8 & $9.0(5.5)$ & 381603 \\
\hline
\end{tabular}

*Defined as first biopsy identified in database. When multiple diagnoses were present, the most severe one was selected.

tCalculated after exclusion of first two years of follow-up. 


\begin{tabular}{|c|c|c|c|c|c|c|c|c|c|}
\hline \multirow[b]{2}{*}{ Mucosal status at baseline } & \multicolumn{3}{|c|}{ Cardia gastric cancer } & \multicolumn{3}{|c|}{ Non-cardia gastric cancer } & \multicolumn{3}{|c|}{ All gastric cancer } \\
\hline & $\begin{array}{l}\text { No of } \\
\text { observed } \\
\text { casest }\end{array}$ & $\begin{array}{l}\text { Crude } \\
\text { incidence } \\
\text { rateł }\end{array}$ & $\operatorname{SIR}(95 \% \mathrm{CI}) \S$ & $\begin{array}{l}\text { No of } \\
\text { observed } \\
\text { casest }\end{array}$ & $\begin{array}{l}\text { Crude } \\
\text { incidence } \\
\text { rate } \neq\end{array}$ & SIR $(95 \% \mathrm{CI}) \S$ & $\begin{array}{l}\text { No of } \\
\text { observed } \\
\text { casest }\end{array}$ & $\begin{array}{l}\text { Crude } \\
\text { incidence } \\
\text { rateł }\end{array}$ & SIR $(95 \% \mathrm{CI}) \S$ \\
\hline \multicolumn{10}{|l|}{ Correa's cascade: } \\
\hline Normal & 24 & 3.9 & $1.0(0.7$ to 1.5$)$ & 97 & 15.6 & $1.1(0.9$ to 1.3$)$ & 121 & 19.5 & 1.0 (0.9 to 1.3$)$ \\
\hline Minor mucosal change & 5 & 5.2 & $1.0(0.3$ to 2.3$)$ & 35 & 36.7 & 1.6 (1.1 to 2.3$)$ & 40 & 41.9 & 1.5 (1.1 to 2.0$)$ \\
\hline Gastritis & 120 & 8.2 & $1.3(1.1$ to 1.6$)$ & 744 & 50.8 & 1.9 (1.8 to 2.1$)$ & 864 & 59.0 & 1.8 (1.7 to 1.9$)$ \\
\hline Atrophic gastritis & 12 & 10.3 & $1.6(0.8$ to 2.8$)$ & 104 & 90.0 & $3.0(2.5$ to 3.7$)$ & 116 & 100.4 & $2.8(2.3$ to 3.3$)$ \\
\hline Intestinal metaplasia & 12 & 17.6 & $2.3(1.2$ to 4.0$)$ & 76 & 111.6 & $3.7(2.9$ to 4.6$)$ & 88 & 129.2 & $3.4(2.7$ to 4.2$)$ \\
\hline Dysplasia & 5 & 29.9 & $3.8(1.2$ to 8.8$)$ & 39 & 232.9 & 7.1 (5.1 to 9.8$)$ & 44 & 262.8 & $6.5(4.7$ to 8.7$)$ \\
\hline \multicolumn{10}{|l|}{ Other diagnoses: } \\
\hline $\begin{array}{l}\text { Cellular degeneration/infiltration } \\
\text { or abnormal karyotype }\end{array}$ & 0 & 0 & - & 4 & 65.4 & $2.5(0.7$ to 6.3$)$ & 4 & 65.4 & $2.0(0.5$ to 5.1$)$ \\
\hline Reactive gastropathy & 1 & 2.8 & $0.8(0.1$ to 4.3$)$ & 3 & 8.4 & $0.7(0.1$ to 2.0$)$ & 4 & 11.2 & $0.7(0.2$ to 1.8$)$ \\
\hline Atypia & 10 & 20.7 & 2.7 (1.3 to 4.9) & 76 & 157.5 & 4.5 (3.6 to 5.7$)$ & 86 & 178.2 & $4.2(3.3$ to 5.2$)$ \\
\hline Hyperplasia or hypertrophy & 7 & 8.0 & $1.4(0.6$ to 2.8$)$ & 45 & 51.2 & 2.1 (1.5 to 2.8$)$ & 52 & 59.2 & $1.9(1.4$ to 2.5$)$ \\
\hline $\begin{array}{l}\text { Metaplasia (not intestinal } \\
\text { metaplasia) }\end{array}$ & 6 & 7.3 & $1.0(0.4$ to 2.1$)$ & 97 & 117.7 & $3.6(2.9$ to 4.3$)$ & 103 & 125.0 & 3.1 (2.5 to 3.7$)$ \\
\hline Polyps & 7 & 6.9 & $1.2(0.5$ to 2.5$)$ & 52 & 51.3 & 2.1 (1.6 to 2.8$)$ & 59 & 58.2 & $1.9(1.5$ to 2.5$)$ \\
\hline Benign tumour & 2 & 10.0 & $1.6(0.2$ to 5.9$)$ & 16 & 80.0 & $3.0(1.7$ to 4.8$)$ & 18 & 90.0 & 2.7 (1.6 to 4.3$)$ \\
\hline \multicolumn{10}{|c|}{$\begin{array}{l}\text { *Defined as first biopsy identified in database. When multiple diagnoses were present, the most severe one was selected. } \\
\text { †First two years of observation and corresponding events were excluded. } \\
\ddagger \text { Per } 100000 \text { person years. } \\
\S 0 \text { bserved to expected number of gastric cancer cases, based on age (five year strata), sex, and calendar year (five year strata) specific in }\end{array}$} \\
\hline
\end{tabular}

along with crude incidence rates and standardised incidence ratios. The annual incidence of total gastric cancer in the normal group was $19.5 \times 10^{-5}$ ( $95 \%$ confidence interval 16.2 to 23.3), which corresponds to the expected incidence in the Swedish population matched on age, sex, and calendar period (standardised incidence ratio 1.0). Among those with chronic non-atrophic gastritis, the annual incidence was $59.0 \times 10^{-5}$ (55.2 to 63.1), corresponding to an $80 \%$ excess in relation to the matched Swedish population (standardised incidence ratio 1.8). The annual incidence increased monotonically with each step in Correa's cascade, up to $262.8 \times 10^{-5}$ (190.9 to 352.8 ) in the dysplasia group. With such an incidence, approximately 1 in 19 people will get gastric cancer within 20 years. Compared with the matched Swedish population, this is a $550 \%$ excess (standardised incidence ratio 6.5). As expected, the excess in relation to the matched Swedish population was greater for non-cardia gastric cancer (in the dysplasia group more than $610 \%$ ), but more moderate for cardia gastric cancer (more than 280\%). When we further stratified by sex, we observed a similar excess incidence of gastric cancer among both males and females (data not shown).

The Nelson-Aalen cumulative incidence plot shows the temporal pattern of total gastric cancer occurrence by mucosal histology group (fig 2). Please note that time 0 in the plot corresponds to day 730 after the initial gastric biopsy. The curves fanned out as expected in the first year, and the increment appears to have been steady throughout the follow-up period in all groups. However, small remaining numbers after censoring in some groups (particularly the intestinal metaplasia group) led to exaggerated increments for new cases of gastric cancer towards the end. The most pronounced gaps seem to have been between non-atrophic gastritis and atrophic gastritis and between intestinal metaplasia and dysplasia. Also, except for intestinal metaplasia-dysplasia and normal-minor change differences (which diminished artifactually towards the end), the gaps kept widening throughout the entire follow-up period.

For the other diagnoses, an excess incidence for gastric cancer was observed in all other lesion groups except the reactive gastropathy group. The top three were atypia (with total gastric cancer as outcome, standardised incidence ratio 4.2), metaplasia (3.1), and benign tumour (2.7). The excess risks were mainly confined to non-cardia gastric cancer, but patients with atypia also had a 2.7-fold increased incidence of cardia gastric cancer (table 2).

Table 3 shows adjusted hazard ratios for the association between gastric mucosal histology groups and occurrence of gastric cancer using Cox regression, with the normal group as reference. The hazards for non-cardia gastric cancer associated with having non-atrophic gastritis and dysplasia were, respectively, 2.8-fold and 12.1-fold increased. Less conspicuous excesses, but still substantial and statistically significant, were also seen for cardia cancer.

For 55621 patients with data on repeat endoscopy (first two years of follow-up excluded), the standardised incidence ratio levels were determined by both baseline mucosal status and subsequent histological change (fig 3). In general the pattern was consistent with the trend observed in the total cohort-standardised incidence ratios increased with more advanced baseline mucosal lesions. Furthermore, within each baseline mucosal status group, progression was consistently associated with a higher incidence of non-cardia gastric cancer risk than "no change," and no change was likewise consistently associated with a higher incidence than regression. For those with a regression of mucosal changes, statistically significant standardised incidence ratios were only observed in the dysplasia group. 


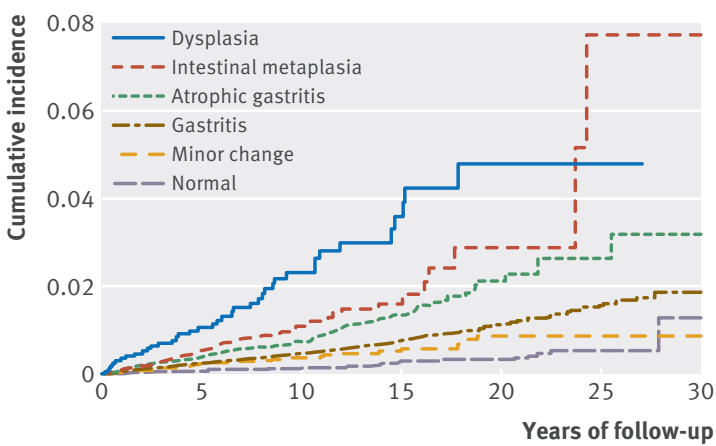

Fig 2 | Cumulative incidence of gastric cancer among patients with different baseline diagnoses. First two years of follow-up excluded

\section{Discussion}

In Sweden, despite the absence of an official guideline, gastroscopy was usually undertaken for upper abdominal symptoms (for example, dyspepsia, pain), gastrointestinal bleeding, suspected reflux (severe heart burn), unexplained iron deficiency anaemia, or malabsorption/ unexplained weight loss. This practice is similar to that of other Western countries ${ }^{2021}$ but can vary by calendar year, age of the patient, and healthcare level. During the studied period there were no widespread and generally accepted guidelines for taking biopsy samples, so the procedure may have differed by time but always included sampling from visible abnormalities. Our observed incidence rates predict that within 20 years after gastroscopy, gastric cancer will develop in approximately 1 in 256 people with normal mucosa, 1 in 85 with gastritis, 1 in 50 with atrophic gastritis, 1 in 39 with intestinal metaplasia, and 1 in 19 with dysplasia. The increased incidence was stable throughout the follow-up period, and the gaps between cumulative incidence curves grew continuously. Interestingly, if repeated biopsy showed a change-upward or (more noteworthy) downward in the Correa's cascade-compared with the initial grouping, this seemed to have prognostic significance.

\section{Comparison to other studies}

To the best of our knowledge, this is the largest follow-up study to date among patients with gastric precancerous mucosal lesions-both for sample size and length of follow-up. The structure of Swedish healthcare essentially

\begin{tabular}{|c|c|c|c|}
\hline \multirow[b]{2}{*}{$\begin{array}{l}\text { Mucosal status at } \\
\text { baseline* }\end{array}$} & \multicolumn{3}{|c|}{ Hazard ratio $(95 \% \mathrm{Cl}) \dagger$} \\
\hline & $\begin{array}{l}\text { Cardia gastric } \\
\text { cancer }\end{array}$ & $\begin{array}{l}\text { Non-cardia gastric } \\
\text { cancer }\end{array}$ & $\begin{array}{l}\text { All gastric } \\
\text { cancer }\end{array}$ \\
\hline Normal & Reference & Reference & Reference \\
\hline Minor mucosal change & 1.1 (0.4 to 2.9 ) & 1.9 (1.3 to 2.8$)$ & 1.8 (1.2 to 2.5$)$ \\
\hline Gastritis & 1.8 (1.2 to 2.9$)$ & 2.8 (2.3 to 3.5$)$ & $2.6(2.2$ to 3.2$)$ \\
\hline Atrophic gastritis & 2.4 (1.1 to 4.8$)$ & $5.0(3.8$ to 6.7$)$ & 4.5 (3.5 to 5.8$)$ \\
\hline Intestinal metaplasia & $4.7(2.3$ to 9.5$)$ & $6.5(4.8$ to 8.9$)$ & $6.2(4.7$ to 8.2$)$ \\
\hline Dysplasia & 6.0 (2.3 to 15.9$)$ & 12.1 (8.3 to 17.6$)$ & $10.9(7.7$ to 15.4$)$ \\
\hline
\end{tabular}

enabled us to include all patients with gastric biopsy. Multiple precise linkages to largely complete high quality national registers-using the national registration numbers as unique identifiers-permitted complete follow-up for cancer outcomes as well as for censoring events. The findings for atrophic gastritis and intestinal metaplasia are principally in agreement with those in another similar large scale follow-up study from Holland ${ }^{8}$ and thus should be generalisable at least to patients undergoing endoscopy in north western Europe. The incidence that we observed among patients with dysplasia, however, was only a fraction of that reported in the Dutch study.

As opposed to the previous study, ${ }^{8}$ our study focused on long term risks; we applied a two year lag time, during which a large peak in incidence was observed. This peak is mainly the result of selection bias-that is, the outcome has an effect on the probability of being examined and thus recruited. Therefore, most of the gastric cancers diagnosed in the first two years were likely present-and overlooked-at the initial investigation or were in such an imminent precancerous stage that they were already symptomatic. Although dysplasia, and particularly severe dysplasia, ${ }^{8}$ is a powerful signal that a gastric cancer may be present (and should heighten clinical observation and prompt repeated endoscopy ${ }^{811}$ ) the concentration of cases in the first two years may blur assessments of the long term prognosis. After two years the peak was passed, so our present data do not include the initially overlooked prevalent cases. This is probably the principal reason for the discrepancy between studies for excess incidence of gastric cancer after a diagnosis of dysplasia (less advanced lesions were much less indicative of a prevalent cancer). None the less, throughout the 15-20 years of follow-up, during which we had sufficient power to monitor the long term progression, patients with dysplasia remained at substantially increased risk. Whether this increase merits institution of scheduled surveillance programmes and, if so, how often and in which ages, must be subject to careful cost-benefit deliberations. Such analysis should ideally provide estimated costs; number of cases of gastric cancer diagnosed and deaths prevented; life years, or quality adjusted life years gained, or both; and incremental cost effectiveness ratio, in association with different screening strategies defined by a combination of, for example, starting age, screening frequency, and risk stratums.

Our study also quantified the risks of gastric cancer among patients with some other gastric mucosal findings. The incidence of gastric cancer among patients with atypia was 4.2-fold higher than in the matched general population. The excess was numerically beyond that observed among patients with intestinal metaplasia. This might be partly attributed to the ongoing debate on the definitions of atypia and dysplasia. ${ }^{22} 23$ Histologically, atypia refers to the presence of cells with cellular abnormalities following rapid cell multiplication under a damaging stimulus. In the case of persistent cellular atypia, typically in the background of thickened epithelium (metaplasia), the term dysplasia should be applied. However, under microscopy it can be challenging to distinguish atypia from dysplasia. This is especially true for endoscopic biopsy samples 
Baseline exposure group

Dysplasia

No change

Regression

Intestinal metaplasia

Progression

No change

Regression

Atrophic gastritis

Progression

No change

Regression

Gastritis

Progression

No change

Regression

Normal or minor mucosal changes

Progression

No change
Standardised incidence ratio $(95 \% \mathrm{Cl})$

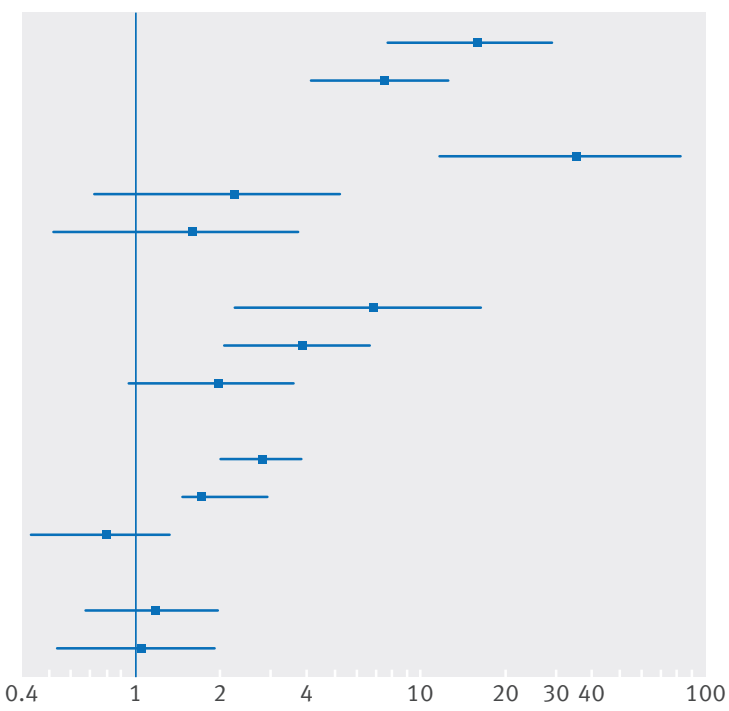

Fig 3 Standardised incidence ratios (95\% confidence intervals) for non-cardia gastric cancer in patients with multiple biopsy records $(n=55621)$, categorised by baseline group and following changing pattern. First two years of observation and corresponding events were excluded. Baseline defined as first biopsy identified in database. When multiple diagnoses were present, the most severe one was selected

where architectural changes are difficult to assess owing to poor tissue orientation and limited amount of material. ${ }^{24}$ Moreover, previous histological studies found that the strictly defined atypia indeed commonly co-occurs (49$78 \%$ ) with gastric cancer. ${ }^{2526}$ Such atypia is therefore considered to be a neoplastic lesion and it has been renamed as "pit dysplasia." Consistent with previous investigations, benign tumours, ${ }^{27}$ non-intestinal metaplasia, polyps, ${ }^{2228}$ and hyperplasia ${ }^{29}$ were all associated with an increased risk of gastric cancer, although the excesses were moderate. Conversely, for reactive gastropathy (an abnormality in the stomach caused by chemical injury), no excess risk for gastric cancer was noted. ${ }^{30}$

\section{Limitations of this study}

The study was limited to patients who underwent gastroscopy with biopsy for clinical indications. This group may differ from the general population in several ways, and our findings cannot be readily generalised to non-patients (or healthy people who undergo screening). On the other hand, only patients presently undergo gastroscopy, and in the study we aimed to create a basis for predictions for this particular group. The fact that virtually all patients who undergo biopsy in Sweden were included in the cohort vouches for reasonable external validity of this group. The standardised incidence ratio for gastric cancer among people with normal mucosa was 1.0, which contradicts a strong link between lifestyle or genetic determinants for gastroscopic examinations and future risk of gastric cancer.

Another caveat to consider is sampling error. Since the studied precancerous conditions tend to be patchy lesions, there is always a risk for false negative results. ${ }^{25}$ It was not possible to obtain detailed information about the subsite being biopsied, and when gastroscopy was

repeated, whether the biopsy samples were taken from the same mucosal area. Inability to capture the stomach's most advanced lesion leads to some overestimation of the incidence of gastric cancer in groups classified as having less advanced lesions, and an underestimation of the range from the lowest to the highest incidence. As already noted, however, the incidence in the normal group was not very different from that expected in the matched population, and a change downward in Correa's cascade proved to be followed by a lower incidence of gastric cancer. These reassuring observations suggest that our results were not unduly affected by sampling error, and also that true reversion of Correa's cascade might be possible. The latter conclusion is at odds with the literature. ${ }^{31-33}$ In addition, since all biopsy samples were taken in routine healthcare and were assessed by many different pathologists, inter-observer and intra-observer variation was a reality, despite the fact that the practice guidelines for the standardised disease diagnosis were set up by the Swedish Society for Pathology. Also, given the prospective design and the two year latency, which essentially removed all prevalent cases of gastric cancer, the misclassification was non-differential for the gastric cancer outcome. The resulting error may have led to conservative estimates of the differences in gastric cancer rate between mucosal histology groups.

To fully understand the pattern of progression, our cohort should ideally have been followed with scheduled and frequent examinations. The lack of close surveillance of the gastric mucosa and the unbalanced distribution of repeat endoscopies in the present study prohibit conclusions about the course and timing of progression through Correa's cascade. However, data from the subset of participants with more than one endoscopy suggested that the observed pattern of change did indeed have prognostic implications.

Finally, we did not have information on factors such as family history of gastric cancer ${ }^{34}$ and environmental or lifestyle exposures, ${ }^{35}$ which could potentially be used to further risk stratify patients. We also suspect that the presence or absence of $H$ pylori was not consistently reported. Future plans for our stomach biopsy cohort include extending the linkage to a multigeneration register to identify a family history of cancer. Taking advantage of existing tissue blocks, which are archived long term at the pathology departments, we will also measure smoking status by immunohistochemical quantitation of related DNA adducts. Furthermore, with our recently developed methods for microbial DNA analysis, ${ }^{36-38}$ we believe that this cohort can offer unique opportunities to expand current knowledge about carcinogenicity-driving specificities of colonising $H$ pylori strains, as well as the role of microbe-environment interactions. Future studies in other settings might consider the collection of additional information on detailed lifestyle factors and other biomarkers to facilitate further risk stratification.

\section{Conclusion}

Our results in a low risk Western patient population show that all stages of Correa's cascade predict an incidence of gastric cancer above that of the general 
population. The observed incidence approximately translates into a 20 year risk of 1 in 85 with gastritis, 1 in 50 with atrophic gastritis, 1 in 39 with intestinal metaplasia, and 1 in 19 with dysplasia among patients who undergo gastroscopy with biopsy for clinical indications. The quantified absolute risks, along with future studies on other risk stratification factors and cost-benefit analyses, should guide the development of surveillance policies.

Contributors: HS was involved in the study design, data collection, analysis and interpretation of the data, and writing the manuscript. IGE was involved in data collection and writing the manuscript. ZZ was involved in conception of the study, data analysis, interpretation of the data, and revision of the manuscript. JE was involved in interpretation of the data and revision of the manuscript. ON and WY were involved in conception of the study, study design, interpretation of the data, and writing the manuscript. All authors revised the article critically for important intellectual content and gave final approval of the version to be published. WY is the guarantor.

Funding: This study was supported by the Swedish Research Council (grant No 2011-3182) and Cancerfonden (grant No 2013-798). HS was partly supported by a scholarship from the China Scholarship Council, and IGE is partly supported by a scholarship from the Karolinska Institutet MD/ $\mathrm{PhD}$ programme. The funders had no role in the conduct of this research. Competing interests: All authors have completed the ICMIE uniform disclosure form at www.icmje.org/coi_disclosure.pdf and declare: no support from any organisation for the submitted work; no financial relationships with any organisations that might have an interest in the submitted work in the previous three years; no other relationships or activities that could appear to have influenced the submitted work. Ethical approval: This study was approved by the regional ethical review board in Stockholm (2010-819-31-3; 2013-1244-32).

Data sharing: No additional data available.

Transparency: The lead author (WY) affirms that this manuscript is an honest, accurate, and transparent account of the study being reported; that no important aspects of the study have been omitted; and that any discrepancies from the study as planned (and, if relevant, registered) have been explained.

This is an Open Access article distributed in accordance with the Creative Commons Attribution Non Commercial (CC BY-NC 4.0) license, which permits others to distribute, remix, adapt, build upon this work non-commercially, and license their derivative works on different terms, provided the original work is properly cited and the use is noncommercial. See: http://creativecommons.org/licenses/by-nc/4.0/.

1 Kapadia CR. Gastric atrophy, metaplasia, and dysplasia: a clinical perspective. J Clin Gastroenterol 2003;36(5 Suppl):S29-36, S61-2.

2 Correa P. Human gastric carcinogenesis: a multistep and multifactorial process-first American Cancer Society award lecture on cancer epidemiology and prevention. Cancer Res 1992;52:6735-40.

3 Lauren P. The two histological main types of gastric carcinoma: diffuse and so-called intestinal-type carcinoma. An attempt at a histo-clinical classification. Acta Pathol Microbiol Scand 1965;64:31-49.

4 IARC working group on the evaluation of carcinogenic risks to humans: some industrial chemicals. Lyon, 15-22 February 1994. IARC Monogr Eval Carcinog Risks Hum 1994;60:1-560.

5 Konturek PC, Konturek SJ, Brzozowski T. Helicobacter pylori infection in gastric cancerogenesis. J Physiol Pharmacol 2009;60:3-21.

6 Song H, Michel A, Nyren O, et al. A CagA-independent cluster of antigens related to the risk of noncardia gastric cancer: associations between Helicobacter pylori antibodies and gastric adenocarcinoma explored by multiplex serology. Int / Cancer 2014:134:2942-50.

7 Sipponen P, Maaroos HI. Chronic gastritis. Scand I Gastroenterol 2015;50:657-67.

8 De Vries AC, van Grieken NC, Looman CW, et al. Gastric cancer risk in patients with premalignant gastric lesions: a nationwide cohort study in the Netherlands. Gastroenterology 2008;134:945-52.

9 Asfeldt AM, Steigen SE, Lochen ML, et al. The natural course of Helicobacter pylori infection on endoscopic findings in a population during 17 years of follow-up: the Sorreisa gastrointestinal disorder study. Eur J Epidemiol 2009;24:649-58.

10 Gonzalez CA, Pardo ML, Liso JM, et al. Gastric cancer occurrence in preneoplastic lesions: a long-term follow-up in a high-risk area in Spain. Int) Cancer 2010;127:2654-60.

11 Dinis-Ribeiro M, Areia M, de Vries AC, Marcos-Pinto R, Monteiro-Soares M, O'Connor A, et al. Management of precancerous conditions and lesions in the stomach (MAPS): guideline from the European Society of Gastrointestinal Endoscopy (ESGE), European Helicobacter Study Group (EHSG), European Society of Pathology (ESP), and the Sociedade Portuguesa de Endoscopia Digestiva (SPED). Endoscopy 2012;44:74-94.
12 Whiting JL, Sigurdsson A, Rowlands DC, et al. The long term results of endoscopic surveillance of premalignant gastric lesions. Gut 2002:50:378-81.

13 Cancer incidence in Sweden 2013. Stockholm: National Board of Health and Welfare, 2015. www.socialstyrelsen.se/ publikationer2014/2014-12-10.

14 Anderson WF, Camargo MC, Fraumeni JF Jr, et al. Age-specific trends in incidence of noncardia gastric cancer in US adults. JAMA 2010;303:1723-8.

15 Song H, Held M, Sandin S, et al. Increase in the prevalence of atrophic gastritis among adults Age 35 to 44 years old in northern Sweden between 1990 and 2009. Clin Gastroenterol Hepatol 2015; published online 6 April.

16 Côté RA. Systematized nomenclature of medicine (SNOMED), 2nd ed. College of American Pathologists, 1979.

17 Ekstrom AM, Signorello LB, Hansson LE, et al. Evaluating gastric cancer misclassification: a potential explanation for the rise in cardia cancer incidence. J Natl Cancer Inst 1999;91:786-90.

18 Ludvigsson JF, Andersson E, Ekbom A, et al. External review and validation of the Swedish national inpatient register. BMC Public Health 2011;11:450.

19 Hansson LE, Nyren O, Hsing AW, et al. The risk of stomach cancer in patients with gastric or duodenal ulcer disease. N Engl I Med 1996;335:242-9

20 Axon ATR, Bell GD, Jones RH, et al. Guidelines on appropriate indications for upper gastrointestinal endoscopy. BMJ 1995;310:853-6.

21 Early DS, Ben-Menachem T, Decker GA, et al. Appropriate use of Gl endoscopy. Gastrointest Endosc 2012;75:1127-31.

22 Zwick A, Munir M, Ryan CK, et al. Gastric adenocarcinoma and dysplasia in fundic gland polyps of a patient with attenuated adenomatous polyposis coli. Gastroenterology 1997;113:659-63.

23 Sanfrancesco J, Jones JS, Hansel DE. Diagnostically challenging cases: what are atypia and dysplasia? Urol Clin North Am 2013;40:281-93.

24 Kelly PJ, Lauwers GY. Gastric polyps and dysplasia. Diagn Histopathol 2001;17:50-61.

25 Agoston AT, Odze RD. Evidence that gastric pit dysplasia-like atypia is a neoplastic precursor lesion. Human Pathol 2014;45:446-55.

26 Kaise M, Kimura R, Nomura K, et al. Accuracy and concordance of endocytoscopic atypia for the diagnosis of gastric cancer. Endoscopy 2014:46:827-32

27 Antonioli DA. Precursors of gastric carcinoma: a critical review with a brief description of early (curable) gastric cancer. Human Pathol 1994:25:994-1005

28 Ginsberg GG, Al-Kawas FH, Fleischer DE, et al. Gastric polyps: relationship of size and histology to cancer risk. Am J Gastroenterol 1996;91:714-7.

29 Goldstone AR, Quirke P, Dixon MF. Helicobacter pylori infection and gastric cancer. J Pathol 1996:179:129-37.

30 Genta RM. Differential diagnosis of reactive gastropathy. Semin Diagn Pathol 2005;22:273-83.

31 Satoh K, Kimura K, Takimoto T, et al. A follow-up study of atrophic gastritis and intestinal metaplasia after eradication of Helicobacter pylori. Helicobacter 1998;3:236-40

32 Wang J, Xu LJ, Shi RH, et al. Gastric atrophy and intestinal metaplasia before and after Helicobacter pylori eradication: a meta-analysis. Digestion 2011;83:253-60.

33 Annibale B, Aprile MR, D’Ambra G, et al. Cure of Helicobacter pylori infection in atrophic body gastritis patients does not improve mucosal atrophy but reduces hypergastrinemia and its related effects on body ECL-cell hyperplasia. Aliment Pharmacol Ther 2000;14:625-34

34 El-Omar EM, Oien K, Murray LS, et al. Increased prevalence of precancerous changes in relatives of gastric cancer patients: critical role of H. pylori. Gastroenterology 2000;118:22-30.

35 Ye W, Ekstrom AM, Hansson LE, et al. Tobacco, alcohol and the risk of gastric cancer by sub-site and histologic type. Int J Cancer 1999;83:223-9.

36 Zheng ZL, Advani A, Melefors O, et al. Titration-free massively parallel pyrosequencing using trace amounts of starting material. Nucleic Acids Res 2010;38:e137.

37 Zheng ZL, Advani A, Melefors O, et al. Titration-free 454 sequencing using Y adapters. Nat Protoc 2011:6:1367-76.

38 Zheng Z, Andersson AF, Ye W, et al. A method for metagenomics of Helicobacter pylori from archived formalin-fixed gastric biopsies permitting longitudinal studies of carcinogenic risk. PLoS One 2011;6:e26442.

C BMJ Publishing Group Ltd 2015

Supplementary table: systematised nomenclature of medicine (SNOMED) diagnostic codes used in study

Supplementary figure: standardised incidence ratios of gastric cancer among patients with biopsy results 